\section{Serological Survey of Mumps Immunity Among Healthcare Workers in Connecticut, December 2006-May 2007}

To the Editor-Mumps is a highly contagious acute viral disease transmitted by oral and respiratory secretions. In unvaccinated persons, unilateral or bilateral parotitis occurs in approximately half of patients. The incubation period of the virus is 16-18 days. Although mumps is usually self limited, adults are more likely than children to develop severe symptoms and complications, such as orchitis, aseptic meningitis, and meningoencephalitis. ${ }^{1}$

The 2006-2007 mumps epidemic in the United States involved 6,584 cases of mumps in different midwestern states. Of patients who had a known vaccination status for measles, mumps, and rubella (hereafter collectively referred to as MMR) and who lived in 8 highly affected Midwestern states, $63 \%$ had received 2 doses, $25 \%$ had received 1 dose, and $13 \%$ had received no vaccine. The national incidence of mumps during this resurgence was 2.2 cases per 100,000 people, with the highest incidence among people 18-24 years of age. ${ }^{2}$

In the United States, the Jeryl Lynn strain of mumps vaccine, currently in use, was introduced in 1967. The combination vaccine for MMR was licensed in 1971 but routinely administered only after $1977 .{ }^{3}$ The incidence of mumps began to decline after 1977, when all 1-year-olds were vaccinated. The recommendation for 2 inoculations of the mumps vaccine for children entering school was instituted by the Centers for Disease Control and Prevention in the 1990s. As a result, after 1989, the incidence of mumps decreased further. In 1994, the requirement for entering the public school system was 1 dose of MMR vaccine. In 2001, 2 doses of MMR vaccine became a mandatory requirement. From 2001 through 2003, fewer than 300 cases of mumps were reported in the United States, a decline of more than $99 \%$ from the 185,691 cases in $1968 .{ }^{4}$

Reports of transmission of mumps in healthcare settings are rare; however, during community outbreaks, exposure of unprotected healthcare workers (HCWs) to mumps is common, both in hospital and community settings.

In June 2007, the Advisory Committee on Immunization Practices approved the adult immunization schedule for October 2007 to September 2008. This update tightened requirements for "presumptive evidence of immunity" to mean 2 doses of MMR vaccine, or serologic evidence, or physiciandocumented mumps infection. ${ }^{5}$

Although there were, at the time, no reported cases of mumps in the state of Connecticut, we assessed the baseline mumps serology status of all HCWs who joined the University of Connecticut Health Center, during preplacement evalua tion. Our goals were to immunize susceptible HCWs to pre vent risk of future mumps transmission, to document th proportion of HCWs who were seronegative for mumps whe they joined our institution, and to guide the development $c$ our institution's protection against mumps.

All newly hired HCWs are required to provide evidence $c$ 2 MMR vaccinations (one of the vaccinations must have os curred after 1980). We conducted a cross-sectional stud. from December 2006 to May 2007, that included all HCW who underwent preplacement or immunization screening $\mathrm{c}$ our employee health service clinic. The study was approve by the institutional review board.

A total of 209 employee health records were reviewed du ing the study period. Data were deidentified. Data on agi sex, and vaccination status and/or history were recorded. Spe cific immunoglobulin $\mathrm{G}$ (IgG) antibodies against mumps vi rus were measured using enzyme-linked immunosorbent as says (ELISAs), at the time of the health center visit. Tests wer conducted according to the standard protocol for ELISA: IgG index values of at least 1.10 were considered positiv results; lower levels were considered negative results. Ac cording to previous studies, the ELISA is simple, rapid, an ideally suited to large-scale mumps serosurveys. ${ }^{6}$ The sensi tivity and specificity of mumps IgG antibody testing by ELIS. are $93 \%$ and $87 \%$, respectively.

During the study period, $209 \mathrm{HCWs}$ underwent preplace ment screening; all had received 2 doses of MMR vaccinatior Most (119) of the HCWs were women. Negative antibod titer results were reported for $16(8 \%)$ of the $209 \mathrm{HCWs}$. Th Table presents the distribution of HCWs across different ag groups; there were $108(52 \%)$ aged 20-29 years, and $8 \mathrm{c}$ these had negative antibody titer results. Susceptible HCW (ie, those with negative antibody titer results) received a: MMR booster dose.

The US mumps outbreak occurred because of crowde campus environments that facilitated transmission of respi ratory and oral secretions. During the outbreak, a high pro portion of the individuals had a documented history of MMR vaccine doses. The effectiveness of 1 dose of mump

TABLE. Results of Titers for Immunoglobulin G Antibodic Against Mumps Virus for 209 Healthcare Workers (HCWs) With History of 2 Doses of Vaccine for Measles, Mumps, and Rubell (MMR), by Age Group

\begin{tabular}{lcccc}
\hline Result & $\begin{array}{c}\text { HCWs aged } \\
20-29 \text { years }\end{array}$ & $\begin{array}{c}\text { HCWs aged } \\
30-39 \text { years }\end{array}$ & $\begin{array}{c}\text { HCWs aged } \\
\geqslant 40 \text { years }\end{array}$ & $\begin{array}{c}\text { All } \\
\text { HCWs }\end{array}$ \\
\hline Negative & $8(4)$ & $6(3)$ & $2(1)$ & $16(8)$ \\
Positive & $100(48)$ & $43(21)$ & $50(24)$ & $193(92)$ \\
Total & $108(52)$ & $49(23)$ & $52(25)$ & $209(10 C$ \\
\hline NOTE. Data are no. (\%) of HCWs.
\end{tabular}


vaccine has been reported as approximately $80 \%$, which is considered inadequate to provide population protection. Previous studies have shown that the effectiveness of 2 doses of vaccine is from $88 \%$ to $95 \%{ }^{8,9}$ The estimated herd immunity threshold for mumps ranges from $88 \%$ to $92 \% .^{10}$

Although there was no single explanation for this outbreak, multiple factors may have contributed; these factors include waning immunity, vaccine failure, high population density and high contact rates in colleges, and incomplete vaccineinduced immunity to the wild virus. The relatively advanced age of the majority of infected patients points toward the waning immunity hypothesis. However, more research is needed to study the long-term vaccine effectiveness.

In our study, all the subjects had received 2 doses of MMR vaccine, and yet $16 \mathrm{HCWs}$ were found to be seronegative. In a recent measles outbreak, an unvaccinated HCW became infected in a hospital. Of 64 people with confirmed cases of measles, 17 became infected while visiting the healthcare facility. ${ }^{11}$

A limitation of our study is the small sample size; we did not include all the HCWs employed. Therefore, the results may underestimate the number of susceptible HCWs already employed.

Mumps should be considered a reemerging yet vaccinepreventable disease, with transmission occurring in both healthcare and community settings. Future studies should include all HCWs, to better assess mumps seroprevalence in healthcare institutions. In view of the possible waning immunity, it is essential to carry out periodic serological surveillance and to vaccinate susceptible HCWs.

\section{ACKNOWLEDGMENTS}

Potential conflicts of interest. All authors report no conflicts of interest relevant to this article.

\section{Amir Mohammad, MD, MPH; Marcia Trape, MD, FACP, FACOEM, MRO; Yosef Khan, MBBS, MPH, MACE}

From the Department of Internal Medicine (A.M.) and the Division of Public Health and Population Sciences, Department of Medicine (M.T.), University of Connecticut Health Center and School of Medicine, Farmington, Connecticut; and the Division of Infectious Diseases, Ohio State University, Columbus, Ohio (Y.K.). (Present affiliation: Yale Occupational and Environmental Medicine Program, Department of Internal Medicine, Yale University School of Medicine, New Haven, Connecticut [A.M.].)

Address reprint requests to Amir Mohammad, MD, MPH, Yale Occupational and Environmental Medicine Program, Department of Internal Medicine, Yale University School of Medicine, 135 College Street, Room 396, New Haven, CT 06510 (amir.mohammad@yale.edu).

Presented in part: American Occupational Health Conference; New York, New York; April 13, 2008 (Poster 114).

Infect Control Hosp Epidemiol 2009; 30:202-203

(1) 2008 by The Society for Healthcare Epidemiology of America. All rights reserved. 0899-823X/2009/3002-0018\$15.00. DOI: $10.1086 / 593966$

\section{REFERENCES}

1. Caplan CE. Mumps in the era of vaccines. CMAJ 1999; 160:865-856.

2. Dayan GH, Quinlisk MP, Parker AA, et al. Recent resurgence of mumps in the United States. N Engl J Med 2008; 358:1580-1589.

3. Mumps vaccine. MMWR Morb Mortal Wkly Rep 1977; 26:393-394.

4. Centers for Disease Control and Prevention (CDC). Update: multistate outbreak of mumps-United States, January 1-May 2, 2006. MMWR Morb Mortal Wkly Rep 2006; 55:559-563.

5. Advisory Committee on Immunization Practices. Recommended adult immunization schedule: United States, October 2007-September 2008. Ann Intern Med 2007; 147:725-729.

6. Van den Hof S, Beaumont MTA, Berbers GAM, de Melker HE. Antibodies against mumps in the Netherlands as assessed by indirect ELISA and virus neutralization assay. Epidemiol Infect 2003; 131:703-709.

7. Tischer A, Andrews N, Kafatos G, et al. Standardization of measles, mumps, and rubella assays to enable comparisons of seroprevalence data across 21 European countries and Australia. Epidemiol Infect 2007; 135: 787-797.

8. Schaffzin JK, Pollock L, Schulte C, et al. Effectiveness of mumps vaccine in a summer outbreak. Pediatrics 2007; 120:e862-e868.

9. Harling R, White IM, Ramsay ME, Macsween KF, van den Bosch C. The effectiveness of the mumps component of the MMR vaccine: a case control study. Vaccine 2005; 23:4070-4074.

10. Anderson RM, May RM. Vaccination and herd immunity to infectious diseases. Nature 1985; 318:323-329.

11. Centers for Disease Control and Prevention (CDC). Measles-United States, January 1-April 25, 2008. MMWR Morb Mortal Wkly Rep 2008; 57:494-498.

\section{Reduction of Hospital-Acquired Methicillin- Resistant Staphylococcus aureus Infection by Cohorting Patients in a Dedicated Unit}

To the Editor-One of the risk factors for methicillin-resistant Staphylococcus aureus (MRSA) acquisition is proximity to MRSA-colonized or MRSA-infected patients who are not receiving care that includes isolation precautions. ${ }^{1}$ Increased numbers of preventable adverse events in patients placed under barrier precautions have been reported recently. ${ }^{2-4}$ These factors may adversely affect the nosocomial infection rates and length of hospital stay (LOS) for patients with MRSA infection. We describe our experience creating a dedicated MRSA infection unit and the implementations that helped reduce the rate of hospital-acquired MRSA infection and average LOS in the medical and surgical units at Crouse Hospital (Syracuse, NY).

Crouse Hospital has 506 acute care beds. In 1999, Crouse Hospital had an outbreak of MRSA infection in the intensive care unit (ICU) during which 1 patient died. Patient beds were situated in close proximity to each other and were separated by curtains. To control the outbreak, all patients in the ICU were screened for MRSA; if they tested positive, they were cohorted to one side of the unit, were placed under contact precautions, and were assigned dedicated staff. The 